\title{
15 End of a project
}

At the end of project funding (BMBF, DFC), TTP and IDAT remain in the "estate".

- What must the relinquishing office ensure before IDAT is handed over?

- What must the receiving institution prove before the TTP transfers and deletes its data?

Data protection law is not linked to the funding of research projects. It is therefore irrelevant to the question of how personal data is to be handled at the end of a project whether a particular funding has been terminated. The decisive factor is whether a research project has been completed or discontinued. In this case, as a rule, personal data will no longer be needed and shall therefore be erased. If, on the other hand, a research project continues to be financed with other funds, this would only have an effect on the question at hand if this would lead to a change in the bodies involved.

There may, however, be necessities to further process personal data in both cases. There may be other legal grounds that allow the processing for archiving purposes or quality assurance purposes for the different fields of research. Special retention periods can be found for example in the medicinal products law as well as in medical devices law. However that cannot be answered in general, but has to be assessed on a case-to-case basis. 
Part II of the legal opinions: Detailed Questions on organisational and technical measures

The Deutsche Forschungsgemeinschaft (DFG) has published guidelines for the handling of research data, which states that according to the rules of Cood Scientific Practice, research data should be archived for at least 10 years in the own institution or in a scientifically relevant, supra-regional infrastructure. ${ }^{52}$ However, data protection requirements take precedence over the guidelines. In many cases IDAT will not be necessary in order to review and assess the validity of data and the quality of scientific work which would be the aim of the recommendation of the DFG. To maintain research possibilities it would be possible to delete name and birth date, converting the latter to an age group, and delete the address converting into assignment to an administrative district.

The idea that data must be returned after processing comes from a time when physical media had to be moved from one place to another and there was a legitimate interest in data recuperation. However, if data is not physically moved from one place to another nowadays, but only electronically duplicated, such a legitimate interest in retrieving data rarely still exists. However, if the sponsor or the person responsible for a research project has an obligation to retain certain data that he does not already have, this data would have to be transferred to him. Otherwise, erasure by the TTP would be sufficient on a regular basis.

- What is normal/usual/allowed at the end of a project, if the erasure of the data is not forced by consent/study protocol ... for example in clinical epidemiological projects? Can the TTP switch off the services but continue to store the data without an ongoing project? Under what conditions?

Erasure does not have to be forced explicitly by the wording of a consent form because the duty to erase data derives according to the provisions in Article 17 GDPR anyway.

Data storage is a form of data processing. It does not make a difference in this respect if a specific service is switched on or off. Data may be stored under the same conditions as the previous processing was allowed.

- How long after the end of the project must the storage of a) consents and $b$ ) research data be ensured, unless otherwise specified?

There is no general rule as to how long consent forms or research data must be stored. Consent forms may be stored at least as long as the processing of data continues and the principle of accountability demands that a controller shall be able to prove that a data subject has consented. More concrete provisions can be found in specific laws covering different fields of scientific research.

52 Deutsche Forschungsgemeinschaft, Leitlinien zum Umgang mit Forschungsdaten, Stand 30.09.2015, p. 1; http:// www.dfg.de/download/pdf/foerderung/antragstellung/forschungsdaten/richtlinien_forschungsdaten.pdf. 
- Is it possible to transfer MDAT and/or IDAT to third parties after the end of the project?

- Under what conditions is the transfer of TTP data (Identifying Data [PII], pseudonyms + mappings, consents and withdrawals) to third parties permissible after the end of the project?

- Under what conditions is the transfer of research data to third parties permitted after the end of the project?

MDAT may be transferred if it can be considered to be anonymous according to recital 26 of the GDPR ${ }^{53}$ from the perspective of the receiving third party. In other cases the transfer of MDAT as well as IDAT must be lawful and would require to be covered by consent or another legal basis. There is no systematic difference as to other processing of personal data.

- Who is responsible for checking the suitability of the third party as data trustee/ processor?

The controller is responsible for ensuring the suitability of a processor.

53 See Part I.2.2.2 